\title{
On the Kalman Filtering Formulation for RTK Joint Positioning and Attitude Quaternion Determination
}

\author{
Daniel Medina ${ }^{\dagger *}$, Anja Heßelbarth ${ }^{\dagger}$, Rauno Büscher ${ }^{\dagger}$, Ralf Ziebold ${ }^{\dagger}$ and Jesús García* \\ $\dagger$ Institute of Communications and Navigation, German Aerospace Centre (DLR), Neustrelitz, Germany \\ ${ }^{*}$ Computer Science and Engineering Department, Universidad Carlos III de Madrid, Madrid, Spain
}

\begin{abstract}
Global Navigation Satellite Systems (GNSS) is the cornerstone for positioning and timing applications across land, sea and air. GNSS carrier phase observations can be used to determine the orientation of a vehicle when this is equipped with a setup of multiple GNSS antennas. Attitude determination seeks to find the rotation between two coordinate frames. In this work, we propose a formulation for the joint estimation of the positioning and attitude using quaternion rotations. The estimation is expressed in the recursive Bayesian estimation framework as an Extended Kalman Filter, whose equations and Jacobians matrices are derived. We evaluate the performance of the proposed methodology with actual data collected from a dynamic scenario under the influence of severe multipath effects.
\end{abstract}

Keywords - GNSS; Attitude determination; Extended Kalman Filter; Quaternion rotation; RTK.

\section{INTRODUCTION}

As the prospective autonomous shipping and the autonomous car are called to completely revolutionize transportation systems, Global Navigation Satellite Systems (GNSS) will be playing a fundamental role in this change. Thus, GNSS technology has been under substantial development over the last years, especially with the prominent upgrowth and deployment of the new Galileo and BeiDou constellations. While position estimation is undoubtedly dominated by the usage of GNSS, attitude determination is one of the lesser known applications of GNSS.

Attitude determination is the process of estimating the orientation of a moving rigid body with respect to its environment and it can be considered as a classical problem with a history of more than 50 years. In a multi-antenna GNSS system, one seeks to find the rotation which relates the baseline vectors joining each pair of antenna positions across the body and the ECEF (Earth-centered Earth-fixed) frames. Although GNSS attitude determination provides substantially higher accuracy than other systems, generally based on magnetic effects, its implementation poses some constraints. On one hand, at least a couple of GNSS receivers providing carrier phase are needed. Although receivers capable of providing carrier phase are currently costly, the technology for the production of low-cost receivers is rapidly growing. On the other hand, attitude accuracy is inversely proportional to the separation between the antennas, making this system impractical for small vehicles.

Orientation can be generally represented in three principal forms: Euler angles, rotation matrix and quaternion. Euler angles describe orientation as three consecutive elemental rotations. Quaternion rotation might not be intuitive and definitely difficult to picture, but it has been widely adopted, especially in robotics and computer graphics, given its singularity-free orientation and numerical stability [1]. There is a wide variety of approaches seeking to solve the attitude problem. Generally, the solutions can be classified into two categories: algebraicbased methods for a deterministic solution [2], or optimal methods based on Batch-Least Squares [3]. In this work, the positioning and the attitude determination problems are coupled together and the resulting nonlinear least squares are recursively solved using an Extended Kalman Filter (EKF).

In [4], a general formulation is presented for GNSS attitude determination problems, showing the relation between carrier phase ambiguity resolution and solving the corresponding attitude matrix. Moreover, Teunissen proposed the C-LAMBDA (Constrained Least-squares AMBiguity Decorrelation Adjustment) method, for which the problem of finding the carrier phase integer ambiguities is constrained adding the GNSS compass problem [5]. In his serie of works [6]-[8], Giorgi further studies and enhances the attitude-aided ambiguity search. So far, this methodology has shown the best performance for GNSS-compass using carrier phase observations. More recently, [9] reviews the attitude determination topic, providing an overview on attitude estimation along with an example setup for the multi-antenna system on a vehicle. Nonetheless, an in-depth discussion on how to formulate a recursive Bayesian estimation of a rigid body attitude using multiple GNSS antennas is still missing.

This work presents a formulation for the simultaneous determination of the carrier phase ambiguities, together with the positioning and the attitude quaternion problems for a GNSS multi-antenna system. The resulting system of nonlinear equations is linearized and solved recursively by applying an EKF. Unlike the works from Teunissen and Giorgi, the integer ambiguity search is not constrained but instead the estimation of the float solution becomes constrained with the GNSS-based attitude problem. The performance of the estimated position and attitude solution are assessed using real measurements acquired during a measurement campaign. For the experimentation, a vessel, equipped with three GNSS receivers in an isosceles right triangle configuration, navigates along an inland waterway in a signal-degraded scenario with severe multipath effects.

The rest of the paper is organized as follows. In section 
2 we provide a brief overview of the GNSS carrier phase positioning. Section 3 describes the attitude determination problem and particularly its solution using multi antenna GNSS setup. The section 4 introduces the joint estimation of position and attitude. Moreover, our proposed EKF is derived and it is compared to a conventional modular approach. The measurement setup and the scenario with associated results is shown in section 5. Finally, the section 6 provides a concise discussion of the obtained results together with the summary and outlook for future work.

\section{GNSS Positioning Problem}

The accuracy of GNSS-based attitude determination is directly proportional to the positioning accuracy. Thus, high precision GNSS positioning based on differential carrier phase measurements is mostly employed when aiming to estimate the attitude of a vehicle. Real Time Kinematics (RTK) is a relative positioning procedure, where the unknown position of a moving rover station is determined with respect to a stationary base station of known coordinates. Figure 1 illustrates the working principle. Due to the single-differencing the satellite clock error is completly eliminated, while the satellite orbit error and the atmosphere-related delays can be reduced significantly, depending on the distance between the base and rover positions ( $p_{B}$ and $p_{R}$ respectively). Then, a pivot satellite (hereinafter referred using the superscript " $r$ ") is chosen for double-differencing, cancelling the delays from the receivers clock offsets.

The carrier phase and pseudorange observations from an $\mathrm{i}^{\text {th }}$ satellite observed by the rover receiver is expressed as

$$
\begin{gathered}
\rho_{R}^{i}=\left\|p^{i}-p_{R}\right\|+I^{i}+T^{i}+c\left(d t_{R}-d t^{i}\right)+\varepsilon_{R}^{i} \\
\Phi_{R}^{i}=\underbrace{\left\|p^{i}-p_{R}\right\|}_{u^{i} \cdot p_{R}}-I^{i}+T^{i}+c\left(d t_{R}-d t^{i}\right)+\lambda N_{R}^{i}+\epsilon_{R}^{i}
\end{gathered}
$$

where:

$\rho_{R}^{i}$ is the code observation in meters,

$\Phi_{R}^{i}$ is the phase observation in meters,

$p^{i}$ is the position of the $\mathrm{i}^{\text {th }}$ satellite,

$p_{R}$ is the position of the rover,

$I^{i}$ is the ionospheric error,

$T^{i}$ is the tropospheric error,

$\lambda$ is the carrier wavelength of the signal,

$N^{i}$ is unknown number of cycles between the receiver and the satellite,

$d t_{R}$ is the receiver clock offset at reception time,

$d t^{i}$ is the satellite clock offset at transit time,

$\varepsilon^{i}, \epsilon^{i}$ are the remaining errors for the code and phase observations respectively, $u^{i}$ is the line-of-sight unit vector (horizontal) between the rover and the $\mathrm{i}^{\text {th }}$ satellite positions.

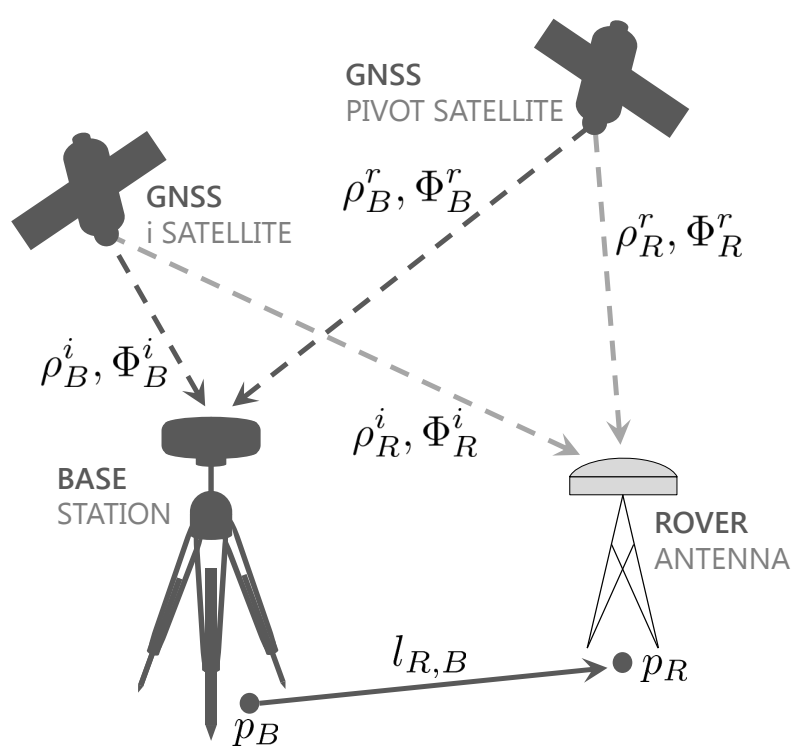

Fig. 1: Illustration of RTK working principle.

As shown in Equation 2, the Euclidean norm between the satellite and receiver positions is often expressed in a linearized form as the product of the line of sight vector $u^{i}$ and the position of the receiver.

The mathematical model of a short-baseline (where the tropospheric and ionospheric delays are neglected) doubledifference carrier phase measurement $D D \Phi$ is given by:

$$
\begin{aligned}
D D \Phi^{i} & =\Phi_{R}^{i}-\Phi_{B}^{i}-\left(\Phi_{R}^{r}-\Phi_{B}^{r}\right) \\
& =u^{i}\left(p_{R}-p_{B}\right)-u^{r}\left(p_{R}-p_{B}\right)+\lambda N^{i, r}+\epsilon^{i, r}
\end{aligned}
$$

The resulting GNSS model is then described as following:

$$
y=A a+G b+\eta
$$

where $y \in \mathbb{R}^{m}$ is the vector is the vector of double differenced code and carrier phase observations, $a \in \mathbb{Z}^{n}$ the vector of carrier phase integer ambiguities, and $b \in \mathbb{R}^{p}$ is the vector containing the rover coordinates and other unknown parameters, such as the atmospheric delay parameters (or in this work, the velocity and the quaternion representing the orientation of the rover). The matrices $A \in \mathbb{R}^{m \times n}$ and $G \in \mathbb{R}^{m \times p}$ relate the observations to the unknown parameters and, lastly, the vector $\eta \in \mathbb{R}^{m}$ is the observation noise vector assumed to follow a zero mean Gaussian distribution.

Solving this problem has been extensively studied in a variety of works [10]-[12]. The parameter estimation is realized by following three steps: float solution, the integer ambiguity estimation and the fixed solution [13]. In our context, we are focusing on the formulation of the EKF to obtain the float solution, extending the regular unknown parameter vector to include the quaternion representing the attitude of the rover. 

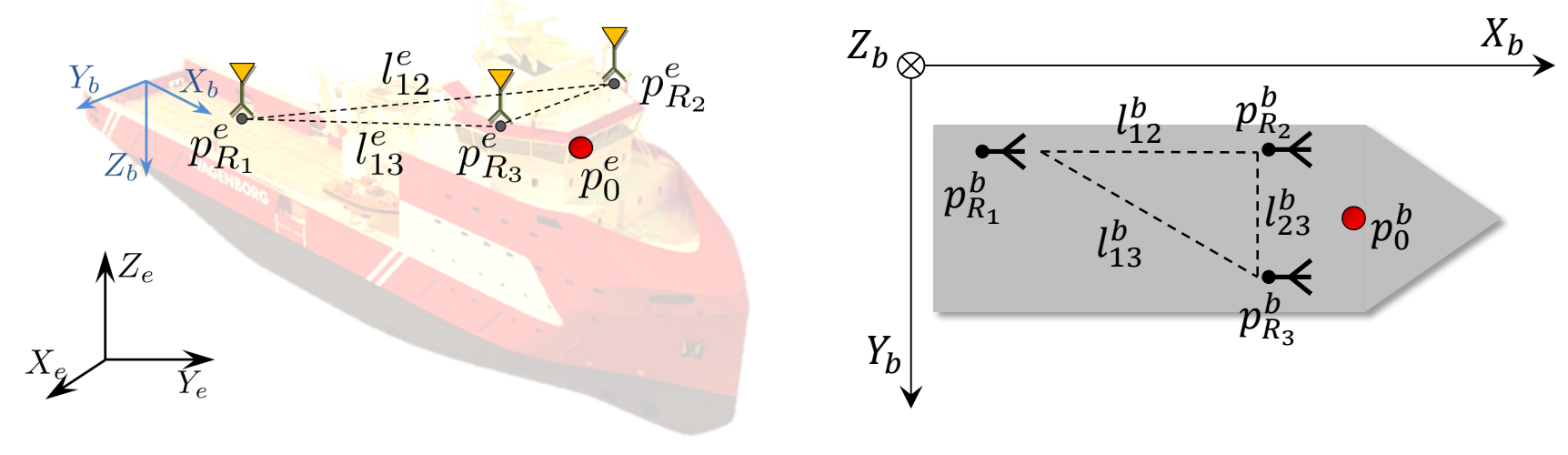

Fig. 2: On the left, baseline description in ECEF. On the right, baseline description in the body frame.

\section{GNSS FOR ATtITUde Determination}

Attitude determination is the process of finding the relationship between two coordinate systems using pairs of vectors belonging to each of these frames. This is considered a classical problem for spacecraft control, whose receivedattention peaked during the late 70s. Finding the optimal attitude solution, given multiple vectors across two frames, was first formulated by Grace Wahba [14] following a least squares scheme:

$$
J\left(C_{b}^{e}\right)=\frac{1}{2} \sum_{k=1}^{N} w_{k}\left\|l_{k}^{e}-C_{b}^{e} l_{k}^{b}\right\|
$$

where $C_{b}^{e}$ is the rotation matrix relating the body to the ECEF (Earth-Center Earth-Fixed) frame, $N=\left(\begin{array}{c}m \\ 2\end{array}\right)$ is the number of pairs of antennas, $l_{k}^{e}, l_{k}^{b}$ correspond to the baseline vector joining the position of two antennas in the ECEF and body frame respectively and $w_{k}$ is the weight of each observation (generally proportional to the baseline length).

For the orientation estimation of a vehicle, at least three GNSS receivers must be arranged in a coplanar configuration. Despite the system in Equation 5 becoming underdetermined, heading information could still be derived using a two-antenna setup. Figure 2 shows an example for the antennas configuration and it will assist illustrating how the baselines and the tracked position are proponed in this work. In guidance and navigation applications, the location of the vehicle's driver is generally tracked. For instance, driver-assistance functionalities for maritime applications requires that the skipper position is estimated. Here, the aforementioned position is noted as $p_{0}$ and it will be the tracked position in our filter as well.

The orientation can generally be represented in three principal forms: Euler angles, rotation matrix and quaternion. Euler angles describe orientation as three consecutive elemental rotations. Although they are conceptually easy to understand, they pose a singularity problem, commonly referred to as gimbal lock, when the pitch angle approaches $\pm 90^{\circ}$. Rotation matrices represent an alternative for attitude parametrization which do not incur any singularity. However, rotation matrices result in difficult renormalization and computational inefficiency (rotation matrices have 9 elements to describe a 3D attitude). Finally, a quaternion is a four dimensional hyper-complex number which is often used to represent the orientation of a rigid body in a 3D space. Although attitude quaternion might not be intuitive and difficult to be visualized, it has been widely adopted, especially in robotics and computer graphics, given its singularity-free orientation and numerical stability [15]. In this work, the quaternion rotation is applied for rotating the baselines from ECEF to the body frame as

$$
l_{i}^{e}=q \otimes l_{i}^{b} \otimes q^{*} .
$$

There are several ways to determine the quaternion depending on the order of its elements (real part first or last), its handedness or the direction of the operation (local-toglobal or global-to-local rotations). This paper follows the Hamilton convention: real part first, right-handed and local-toglobal rotation. For more details on the different quaternion conventions and a comprehensive explanation on quaternion algebra, the authors suggest consulting the work of Solà in [15].

As discussed previously, the accuracy of the attitude estimation is bounded by two factors: the distance between antennas and the positioning precision. In [6], an experimental test is carried out with the aim of relating the heading estimation performance to the baseline length and the number of satellites. Here, we discuss how the angular error of the heading estimation can be characterized following a geometrical approach. Figure 3 depicts the position of two GNSS antennas $p_{1}$ and $p_{2}$ separated by a distance $l$. If there is a positioning error $\delta p$ on the determination of one of the antennas, the heading error $\delta \theta$ can be formulated as

$$
\sin (\delta \theta)=\frac{\delta p}{\sqrt{l^{2}+\delta p^{2}}}
$$

considering that the positioning error is considerably smaller than the baseline length $\left(l^{2}+\delta p^{2}=l^{2}\right)$ and under the smallangle assumption $(\sin (\delta \theta)=\delta \theta)$, the accuracy of the attitude can be described by

$$
\delta \theta \simeq \frac{\delta p}{l}
$$




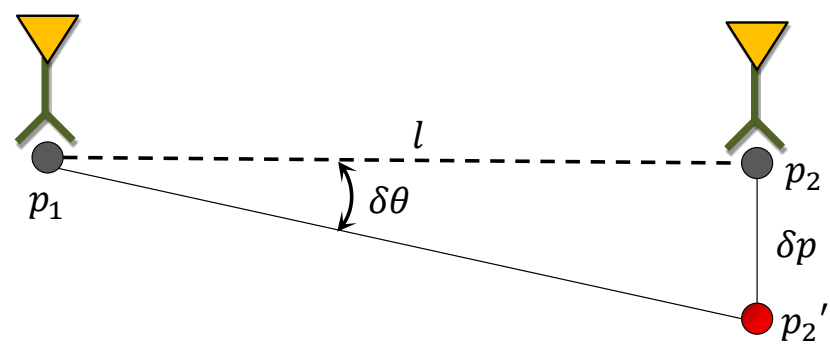

Fig. 3: Sketch regarding the characterization of the heading accuracy. $\delta \theta$ stands for the unknown error on the attitude determination.

This hyphotesis agrees with the results exposed in [8]. In Figure 4, it is shown what would be the expected attitude determination accuracy depending on the length of the baseline and the nominal performance of different positioning techniques (1 meter for code Single Point Positioning, 10 centimeters for Precise Point Positioning and 1 centimeter for RTK).

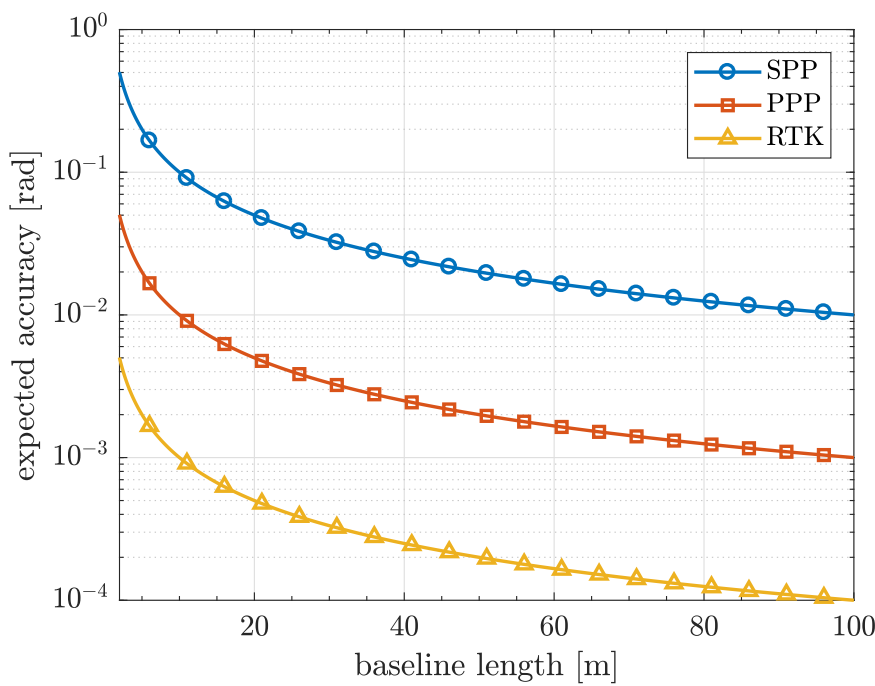

Fig. 4: Theoretical heading accuracy as a function of the baseline length and the nominal accuracy of different positioning techniques.

\section{Joint Positioning And Attitude Estimation}

As aforementioned, the procedure of differential phasebased positioning comprises three steps: float solution, integer ambiguity fixing and state reconstruction or fixed solution [13]. This work focuses on finding the float solution and it presents the derivation of an EKF for the joint estimation of the positioning and heading of a vehicle. The float solution is the phase, in which the ambiguities and the other unknowns are estimated as real numbers. This problem is generally solved using a least squares (LS) estimation, which yields an optimal solution under the assumption of Gaussian noise (hypothesis generally accomplished by GNSS observations, whenever they are not affected by severe atmospheric conditionsor multipath).
EKF is a Bayesian estimator often applied for determining the float solution [16]-[18]. Given its recursive nature, the estimated float solution improves over time (although the filter can be applied in a snapshot modus, becoming equivalent to a regular LS [19]). Following the notation from Equation 4, our system state $x$ is constituted of two set of unknown parameters $: b$ the vehicle dynamics (position, velocity and quaternion orientation) and $a$ the single-differenced ambiguities.

$$
x=\left[\begin{array}{l}
b \\
- \\
a
\end{array}\right]=\left[\begin{array}{l}
p \\
v \\
q \\
a
\end{array}\right]
$$

where

$p$ is the tracked position of the rover in ECEF (which also corresponds to $p_{0}^{e}$ in Figure 2),

$v$ is the velocity in ECEF,

$q$ describes the rotation from body to the ECEF frame.

Kalman filters perform in two steps, prediction-correction, recursively. During the prediction step, the system evolves following a constant-velocity dynamical model. There are handful models to select from, and the filter performance can be enhanced augmenting the prediction with sensors such as inertial measurement units (IMU). With regards to the correction step, the system is updated using the doubledifferenced code and phase observations from the antennas. Equation 3 can be reformulated for the $k$ antenna as:

$$
D D \phi_{k}^{i}=\left(u^{i}-u^{r}\right)\left(p_{R_{k}}-p_{B}\right)+\lambda\left(a_{k}^{i}-a_{k}^{r}\right)+\eta
$$

Let us substitute the position of the antenna $p_{R_{k}}$ by a function of the tracked position $p$ and the known baseline between their positions in the body frame $l_{k}^{b}$

$$
p_{R_{k}}=p+q \otimes l_{k}^{b} \otimes q^{*} .
$$

Equation 10 is then expressed as

$D D \Phi_{n}^{i}=\left(u^{i}-u^{r}\right)\left(p+q \otimes l_{n}^{b} \otimes q^{*}-p_{B}\right)+\lambda\left(a^{i}-a^{r}\right)+\eta$

The EKF update is ruled by the following equations:

$$
\begin{aligned}
& x_{t \mid t}=x_{t \mid t-1}+K_{t}\left(y_{t}-H_{t} x_{t \mid t-1}\right) \\
& P_{t \mid t}=P_{t \mid t-1}-K_{t} H_{t} P_{t \mid t-1} \\
& K_{t}=P_{t \mid t-1} H_{t}^{T}\left(H_{t} P_{t \mid t-1} H_{t}^{T}+R_{t}\right)^{-1}
\end{aligned}
$$

For simplicity, henceforth the subscript $t$ of the recursive estimation will be dropped. The code and phase doubledifferenced observations for each of the $k$ antennas are given as

$$
\begin{gathered}
y_{k}=\left[\begin{array}{c}
D D \Phi_{k} \\
D D \rho_{k}
\end{array}\right], \\
H_{k}=\left[\begin{array}{llll}
G_{k} & 0 & G_{k} J_{q}\left(l_{k}^{b}\right) & A_{k} \\
G_{k} & 0 & G_{k} J_{q}\left(l_{k}^{b}\right) & 0
\end{array}\right]
\end{gathered}
$$

where $J_{q}(l)$ represents the Jacobian of the baseline rotation with respect to the quaternion. The derivation of this operation 


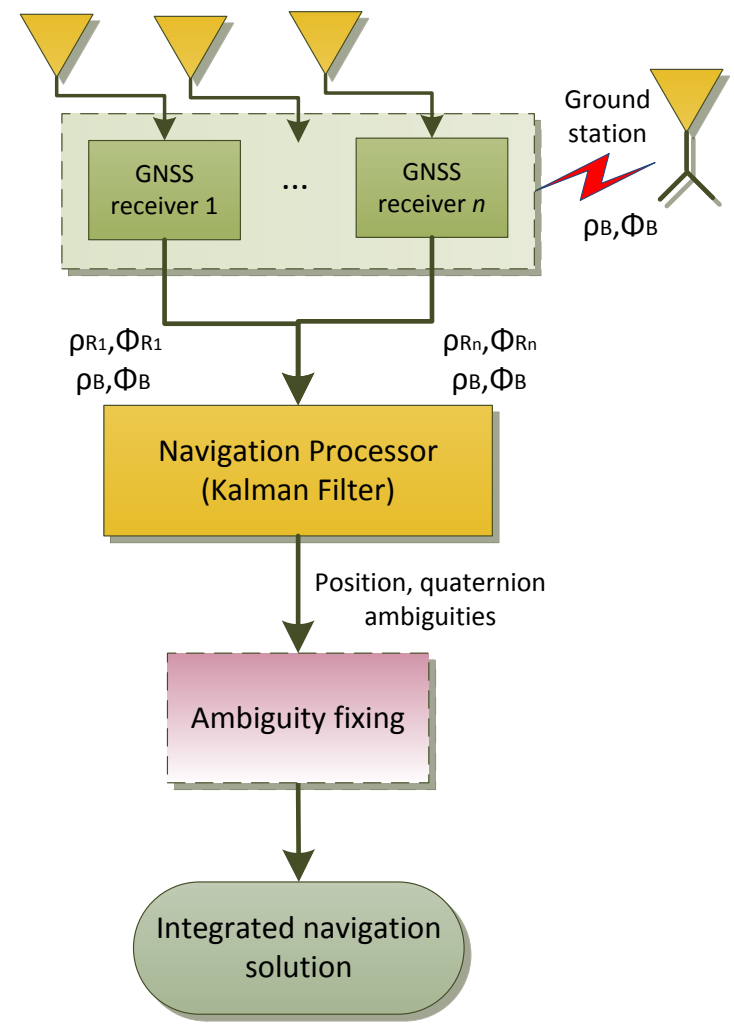

Fig. 5: Our proposal for jointly solving the positioning and heading problem. We will refer to this approach as "Joint EKF"

can be found in [15]. Finally, the measurements from the antennas are stacked in a single vector one underneath the other. The same operation is repeated for the matrix $\mathrm{H}$ :

$$
y=\operatorname{vec}\left(y_{1}, \ldots, y_{k}\right), \quad H=\operatorname{vec}\left(H_{1}, \ldots, H_{k}\right)
$$

The covariance matrix of the observation noise $R$ is obtained by double differencing the diagonal matrix containing the variances of the original non-differenced observations. For more details on the estimation of $R$ and the models used in this work, refer to [20].

The joint estimation of the position and the orientation of a vehicle is not a widespread technique. Instead, a rather simple but effective methodology is followed: the position of the three antennas are independently estimated and then an explicit solution for the attitude is found using memoryless techniques (a survey on these methods can be found in [21]). Figures 5 and 6 provide flow diagrams for the approach introduced in this paper and for the traditional approach respectively. There is an intriguing discussion regarding which of the two methodologies to follow, as both options present different pros and cons. On one hand, our proposal yields some disadvantages, such as the increased complexity of the filter. Also, the non-linearity nature of the attitude problem could drag a poor performance from the linearization implicit in the EKF [21]. Regarding the advantages, the proposed

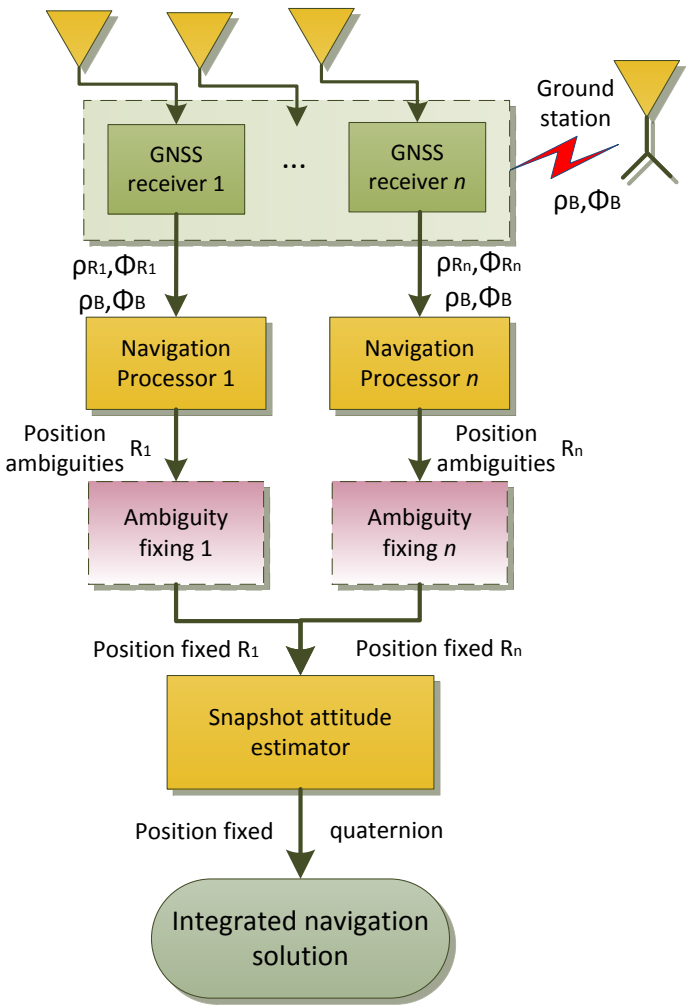

Fig. 6: Approach for parallel track for each of the antenna positions and estimation of the attitude using a memory-less technique. This approach will be refered to as "Modular EKF".

EKF provides an indicator of the determined attitude quality in the form of covariance. Moreover, our system can be easily augmented to use additional sensors, what can largely increase the system performance (especially when high quality gyroscopes are employed). On the other hand, the approach shown in Figure 6 also offers some clear advantages. The system is totally modular and the different tasks- estimation of the antenna positions and the later ambiguity fixing -can be parallelized. Besides, snapshot solutions such as QUEST are able to provide an explicit solution to the Wahba's problem from Equation 5 as they are not as constrained by the nonlinearity of the system as EKFs.

\section{EXPERIMENT AND RESULTS}

The performance of the position and heading determination of the proposed method is evaluated using real observations recorded during the measurement campaign on 16th May 2017 (DOY 208, UTC 12:00-13:30) conducted in Koblenz (Germany) on the Moselle river, in cooperation with the German Federal Waterways and Shipping Administration for the LAESSI (German acronym for Control and Assistance Systems to Enhance the Safety of Navigation in InlandWaterways) project [22]. The data is collected on board of the vessel MS Bingen (Figure 7) at $2 \mathrm{~Hz}$, with an elevation mask of $5^{\circ}$. The equipment consists of three navXperience GNSS antennas connected to three geodetic Javad DELTA receivers. 


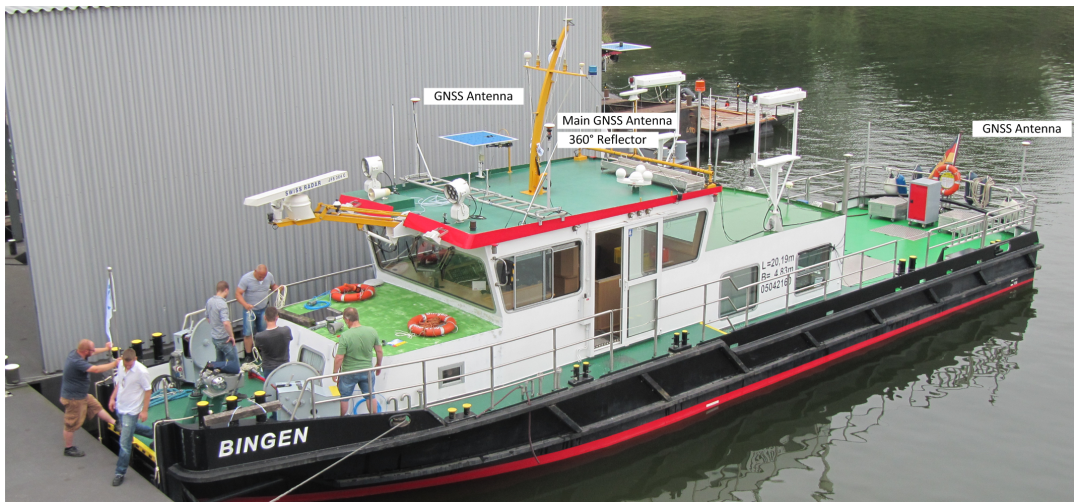

Fig. 7: vessel MS Bingen during the measurement campaign in Koblenz on $16^{\text {th }}$ May, 2017

Although observations from GPS, GLONASS and Galileo are available, for this study only GPS L1 and L2 observations will be used. As it can be seen in Figure 7, the setup for the antennas followed the same scheme as the example of body frame shown in Figure 2. Antennas 1 and 2 are aligned with the axis $X^{b}$, and antennas 2 and 3 are aligned with $Y^{b}$. The antenna positions in the body frame and the corresponding baselines between the antennas is

$$
\begin{aligned}
& p_{R_{1}}^{b}=\left[\begin{array}{lll}
0.57, & -1.98, & -2.09
\end{array}\right], \\
& p_{R_{2}}^{b}=\left[\begin{array}{lll}
3.82, & 1.62, & -4.30
\end{array}\right], \\
& p_{R_{3}}^{b}=\left[\begin{array}{lll}
13.82, & -1.69, & -4.29
\end{array}\right],
\end{aligned}
$$

resulting in baselines lengths of $13.44,13.91$ and 3.32 meters.

Along one hour and a half, the vessel left the harbor and travelled around 8 kilometers in the demonstration area displayed in Figure 9. Due to the presence of four bridges and a waterway lock, severe multipath and none-line-ofsight effects make reliable and continous positioning fairly challenging. The number of GPS satellites tracked and the PDOP (Precision Dilution of Precision) is shown in Figure 8. Throughout the study, there is a minimum of eight satellites locked. However under the bridges and in the vicinity of those, satellite-availability is reduced up to three satellites.

The position and attitude solutions from the proposed approach is compared to a more traditional methodology, in which the position of the antennas are independently estimated and then the attitude is derived using a snapshot method. For the comparison, QUEST [23] is used as the explicit estimator for the attitude solution in the modular EKF. Regarding the ambiguity fixing process, the standard MLAMBDA (Leastsquares AMBiguity Decorrelation Adjustment) method from [24], [25] is applied.

The rate of fixed solution obtention is shown in Table I, comparing the performance of the joint and modular EKF approaches. Both approaches provide a comparable effectiveness, although the joint EKF outperforms the modular concept by $0.70 \%$ (slightly over half a minute of fixed navigation solution). Concerning the attitude determination, Figures 10, 11 depicts the estimated Euler angles obtained with the two

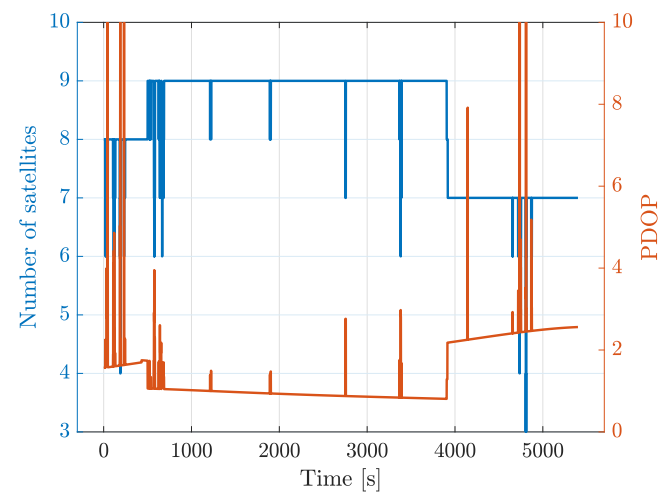

Fig. 8: Number of visible satellites and PDOP.
TABLE I: Percentage of fixed solutions (\%) depending on the number of locked satellites. Comparison between the joint and the modular EKF.

\begin{tabular}{c|cc} 
Number of satellites / Time (\%) & Joint EKF & Modular EKF \\
\hline $9 /(61.2 \%)$ & 82.2 & 82.0 \\
$8 /(9.2 \%)$ & 78.4 & 78.2 \\
$7 /(28.2 \%)$ & 65.7 & 63.9 \\
$6 /(0.9 \%)$ & 2.1 & 2.1 \\
$\leq 5 /(0.5 \%)$ & 0 & 0 \\
\hline Total & 76.1 & 75.4
\end{tabular}

compared approaches. The represented Euler angles follow the aircraft principal axes convention (roll for the longitudinal axis, pitch for the lateral axis and yaw for the normal axis) for relating the body to the local tangent plane NED (NorthEast-Down). In general, EKF is expected to a nominal inferior performance given the non-linearity of the attitude problem. This statement agrees with the histograms shown in Figure 11, where the variances of the EKF solution for the roll and pitch are slightly wider than QUEST (the snapshot attitude solver applied in the modular EKF). Similar behaviour is deduced from the estimation of the heading over time shown in Figure 10. Generally, the estimated heading from both methods is consonant althought EKF appears to misbehave during in some epochs at the end of the measurement campaign (around 5200 s).

\section{Summary AND CONCLUSIONS}

A method for the recursive estimation of the positioning and attitude problems using GNSS carrier phase observations has been proposed in this work. The GNSS community has committed an extensive effort on constraining the integer least squares for an enhanced attitude determination. However, to the best of our knowledge, improving the quality of the float solution for the joint position and heading was yet to be exploited. Our proposed method yields distinct assests. Firstly, additional observation redundancy is obtained by unidtely using the measurements from all the antennas. Besides, the framework for Bayesian estimation provides a covariance as a quality indicator for the attitude solution, what can be 


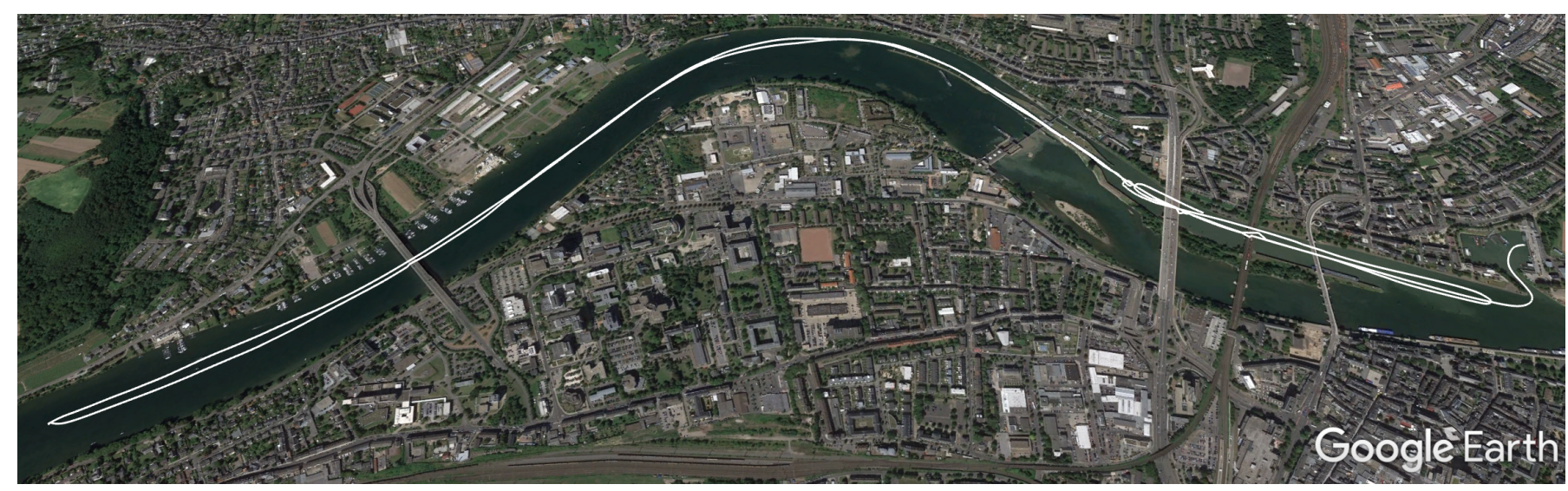

Fig. 9: Measurement area on the river Moselle near Koblenz (Germany). Reference main path (green marks). Detail on the performance of position estimation from the regular multi-constellation WLS (red line) under the central bridge compared to the reference position (green line).

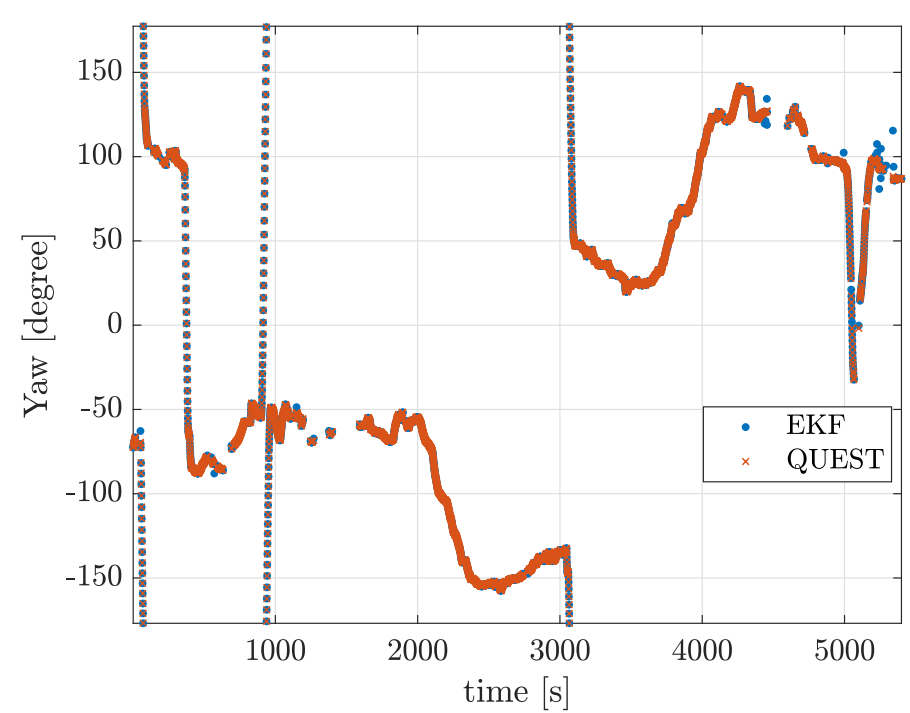

Fig. 10: Heading estimation over time
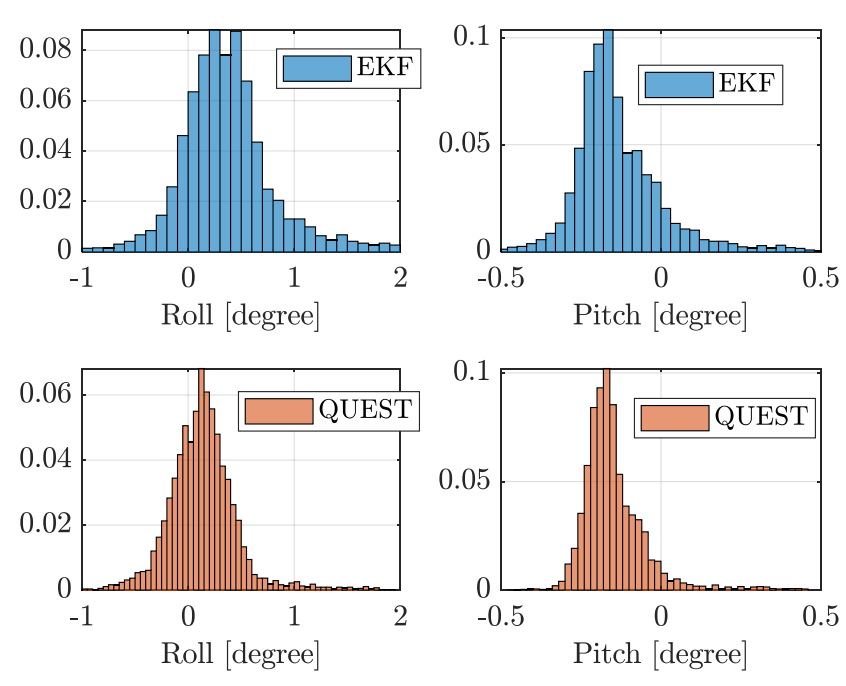

Fig. 11: Histogram of the estimated pitch and roll.

during the correction step in the EKF so that the nonlinearity incompability of the problem gets reduced. Finally, an interesting discussion can be found in [26] with regards to the uncertainty propagation of quaternion and Euclidean states. Unlike the position and velocity, the attitude should not be assumed to be Gaussian-distributed since it lays in the interval $[-\pi, \pi)$. Thus, constructing a filter which handles Gaussian and circular normal distributions is another prospective topic to explore in relation to attitude determination.

\section{ACKNOWLEDGMENT}

The authors would like to thank the partners from LAESSI for their help and collaboration during the measurement campaigns. Also, we highly appreciate the fruitful and endless discussions with Dr. Michailas Romanovas. between the antennas is applied to improve the ambiguity search. Furthermore, certain modifications can be implemented 


\section{REFERENCES}

[1] N. Trawny and S. I. Roumeliotis, "Indirect kalman filter for 3d attitude estimation," University of Minnesota, Dept. of Comp. Sci. \& Eng., Tech. Rep, vol. 2, p. 2005, 2005.

[2] F. L. Markley, "Attitude determination using vector observations: A fast optimal matrix algorithm," 1993.

[3] M. D. Shuster, "Maximum likelihood estimation of spacecraft attitude," J. Astronaut. Sci., vol. 37, pp. 79-88, 1989.

[4] P. Teunissen, "A general multivariate formulation of the multi-antenna gnss attitude determination problem," Artificial Satellites, vol. 42, no. 2, pp. 97-111, 2007.

[5] _ - "The lambda method for the gnss compass," Artificial Satellites, vol. 41, no. 3, pp. 89-103, 2006

[6] G. Giorgi, P. J. Teunissen, S. Verhagen, and P. J. Buist, "Testing a new multivariate gnss carrier phase attitude determination method for remote sensing platforms," Advances in Space Research, vol. 46, no. 2, pp. 118129, 2010.

[7] G. Giorgi and P. Teunissen, "Gnss carrier phase-based attitude determination," in Recent advances in aircraft technology. InTech, 2012, pp. 193-220.

[8] G. Giorgi, P. J. Teunissen, and T. P. Gourlay, "Instantaneous global navigation satellite system (gnss)-based attitude determination for maritime applications," IEEE Journal of oceanic engineering, vol. 37, no. 3, pp. 348-362, 2012.

[9] I. GNSS. (2017) How do you use GNSS to compute the attitude of an object? [Online]. Available: http://www.insidegnss.com/node/5622

[10] P. J. Teunissen, "The least-squares ambiguity decorrelation adjustment: a method for fast gps integer ambiguity estimation," Journal of geodesy, vol. 70, no. 1-2, pp. 65-82, 1995.

[11] R. Odolinski, P. J. Teunissen, and D. Odijk, "Combined bds, galileo, qzss and gps single-frequency rtk," GPS solutions, vol. 19, no. 1, pp. $151-163,2015$.

[12] A. Brack, "Partial ambiguity resolution for reliable gnss positioninga useful tool?" in Aerospace Conference, 2016 IEEE. IEEE, 2016, pp. $1-7$.

[13] P. Joosten and C. Tiberius, "Lambda: Faqs," GPS Solutions, vol. 6, no. 1-2, pp. 109-114, 2002.

[14] G. Wahba, "A least squares estimate of satellite attitude," SIAM review, vol. 7, no. 3, pp. 409-409, 1965

[15] J. Solà, "Quaternion kinematics for the error-state kalman filter," CoRR, vol. abs/1711.02508, 2017. [Online]. Available: http://arxiv.org/abs/1711.02508

[16] C. Eling, L. Klingbeil, and H. Kuhlmann, "Development of an rtk-gps system for precise real-time positioning of lightweight uavs," in Proc. of, 2014.

[17] S. Zhao, X. Cui, F. Guan, and M. Lu, "A kalman filter-based short baseline rtk algorithm for single-frequency combination of gps and bds," Sensors, vol. 14, no. 8, pp. 15 415-15433, 2014.

[18] P. Henkel, M. Iafrancesco, and A. Sperl, "Precise point positioning with multipath estimation," in 2016 IEEE/ION Position, Location and Navigation Symposium (PLANS), April 2016, pp. 144-149.

[19] O. G. Crespillo, D. Medina, J. Skaloud, and M. Meurer, "Tightly coupled gnss/ins integration based on robust m-estimators," in 2018 IEEE/ION Position, Location and Navigation Symposium (PLANS), April 2018.

[20] C. Eling, L. Klingbeil, and H. Kuhlmann, "Real-time single-frequency gps/mems-imu attitude determination of lightweight uavs," Sensors, vol. 15, no. 10, pp. 26212-26235, 2015.

[21] J. L. Crassidis, F. L. Markley, and Y. Cheng, "Survey of nonlinear attitude estimation methods," Journal of guidance, control, and dynamics, vol. 30, no. 1, pp. 12-28, 2007.

[22] C. D. German, Aerospace. (2015) LAESSI control and assistance systems to enhance the safety of navigation in inland waterways. [Online]. Available: http://www.dlr.de/kn/en/desktopdefault.aspx/tabid4307/6939read-45318/

[23] M. Shuster, "Approximate algorithms for fast optimal attitude computation," in Guidance and Control Conference, 1978, p. 1249.

[24] X.-W. Chang, X. Yang, and T. Zhou, "Mlambda: a modified lambda method for integer least-squares estimation," Journal of Geodesy, vol. 79, no. 9, pp. 552-565, 2005.

[25] M. Borno, X.-W. Chang, and X. Xie, "On decorrelationin solving integer least-squares problems for ambiguity determination," Survey review, vol. 46, no. 334, pp. 37-49, 2014.
[26] J. E. Darling and K. J. DeMars, "Uncertainty propagation of correlated quaternion and euclidean states using the gauss-bingham density," Journal of Advances in Information Fusion, vol. 11, no. 2, pp. 1-20, 2016. 